\title{
Comunicación en salud y atención primaria. Retos Y propuestas de solución
}

\author{
Luis Antonio Reyna*
}

\section{Citar este artículo así:}

Reyna LA. Comunicación en salud y atención primaria. Retos y propuestas de solución. Hacia. Promoc. Salud. 2021; 26 (1): 15-16 DOI: 10.17151/hpsal.2021.26.1.2

Desde que se declaró la importancia de los sistemas de salud (SS) basados en Atención Primaria (APS) en 1978, la aplicación de esta premisa teórica sigue siendo un reto, especialmente en Latinoamérica. Entre las razones principales se encuentran: la amplia diversidad de interpretaciones de la APS y la pobre utilización de técnicas de comunicación, por parte del personal de los SS (1). Es decir, las personas que conforman los SS no cuentan con las suficientes competencias para aplicar las bases teóricas de la APS en su quehacer cotidiano, especialmente en el rubro de la comunicación. Esto no es de extrañarse, ya que al menos en México, el perfil profesional predominante en los SS es clínico, particularmente médico; en donde históricamente la APS y la comunicación en salud (CS) no suelen formar parte de los currículos universitarios.

Actualmente continúa la idea de que la CS se limita a las campañas informativas que las instituciones dirigen a la población a través de televisión, radio u otros medios. Sin embargo, la CS es una disciplina que estudia lo relacionado a la información: "cómo y quién la genera, cómo se estructura y se construye al servicio de una institución, cómo es percibida, intercambiada y el conocimiento de su impacto social" (2,19 pX); por lo tanto, la CS es un proceso social, educativo y político que promueve la acción comunitaria en pro de la conciencia pública sobre la salud (3).

Como resultado de lo anterior, se desaprovechan las oportunidades que ofrecen las bases teórico-prácticas de la CS en la estructuración de estrategias para ese primer contacto que tiene la población con los SS y la consecuente asistencia esencial accesible, como se concibe la APS, las cuales pueden ser analizadas y adaptadas en distintos contextos. Desafortunadamente, las estrategias institucionales de CS continúan siendo improvisadas, enfocadas en difundir "instrucciones saludables" o mensajes dispersos centrados en la enfermedad realizadas por personal no especializado, lo que ha limitado las posibilidades de acción de los SS basados en APS.

En respuesta a lo anterior, se han propuesto dos líneas estratégicas convergentes como marco teórico para ser tomado en cuenta por parte de las universidades: la reorientación de la formación de profesionales de la salud, particularmente de los médicos, a través de una educación basada en la comunidad y con un

\footnotetext{
* Médico Salubrista, Doctor en Imagen, Arte, Cultura y Sociedad. Profesor Investigador de Carrera, adscrito al Departamento de Ciencias de la Medicina, de la Universidad de Quintana Roo, México.

Contacto: dr.reynamtz@uqroo.mx (D) orcid.org/0000-0003-2962-4899. Google
}

Hacia promoc. salud., Volumen 26, No.1, enero - junio 2021, págs. 15-16 ISSN: 0121-7577 (Impreso) ISSN: 2462-8425 (En línea) 
enfoque de APS; y la institucionalización de la comunicación en los planes nacionales y académicos de salud pública que incentiven la capacitación e investigación de esta disciplina para el fortalecimiento y motivación del talento humano en los SS $(4,5)$.

De esta forma, a través de la reorientación académica, los sistemas de salud contarían con profesionales capaces de desarrollar procesos comunicativos para la promoción de políticas públicas y procesos sociales, que brinden soluciones a problemáticas de salud global.

\section{Referencios bibliográficos}

1. Organización Panamericana de la Salud, Organización Mundial de la Salud. Renovación de la atención primaria de salud en las Américas: documento de posición de la Organización Panamericana de la Salud/Organización Mundial de la Salud (OPS/OMS). Washington, D.C: OPS; 2007.

2. Dirección General de Promoción de la Salud. Manual de mercadotecnia social en salud. México, DF: Secretaría de Salud, 2010.

3. Choque-Larrauri R. Comunicación y educación para la promoción de la salud. Razón y Palabra. 2005; Vol (Número),1-17. DOI o URL

4. Organización Panamericana de la Salud. La Formación en Medicina Orientada hacia la Atención Primaria de Salud. Washington D.C: OPS; 2008

5. Mosquera-Vásquez M. Comunicación en salud: conceptos, teorías y experiencias. En: Psicología de la salud: Temas actuales de investigación en Latinoamérica. Colombia: ALAPSA; UNINORTE; 2005. p. 209-30. 